\title{
Resistencia, cambio y adaptación en las universidades argentinas: problemas conceptuales y tendencias emergentes en el gobierno y la gestión académica
}

\author{
Claudio Suasnábar \\ Facultad de Humanidades y Ciencias de la Educación, Universidad Nacional de La Plata, Argentina
}

\section{Introducción}

En los últimos años, las universidades nacionales vienen cambiando su fisonomía aceleradamente, produciéndose cambios quizás solamente comparables a los ocurridos durante los años sesenta. Cierto es que las transformaciones en curso vienen marcadas por una profunda acción estatal sobre el conjunto del sistema universitario, y que no siempre ésta fue, o es, respetuosa de la autonomía de estas instituciones. Pero asignar un papel preponderante al Estado en esta coyuntura significa perder de vista los procesos de cambio a nivel interno, que tienen su expresión en la configuración y desarrollo de corrientes de opinión en el seno del cuerpo académico, y en las lógicas de funcionamiento que regulan la producción, reproducción e inter-relación entre grupos y segmentos académicos. Sólo desde esta perspectiva, que intenta tomar en cuenta ambos niveles, es posible adentrarse en un análisis más ajustado sobre el sentido, profundidad y consecuencias de este proceso de transformación.

Planteado de esta forma, la realidad de las universidades nacionales se nos presenta cada vez menos homogénea y nos remite al análisis de casos concretos. Si bien las políticas estatales tienden a estructurar y homogeneizar el sistema sobre la base de nuevos criterios y valores, las características específicas de cada institución y su grado de autonomía marcan las diferencias en el tipo de respuestas, tendiendo con ello a una mayor heterogeneidad. Así, las políticas académicas institucionales son el resultado de estas tendencias contradictorias, que se despliegan en un continuo que va desde acciones meramente adaptativas o reactivas frente a las políticas estatales hasta acciones que podrían marcar líneas de trabajo de proyectos institucionales autónomos.

Sin embargo, y pese a la relevancia que podrían aportar estudios de esta naturaleza, la producción académica en nuestro país se ha enfrentado al menos con dos obstáculos para avanzar en esta dirección. Por un lado, tradicionalmente el área de política , y más en general, de política educativa, ha privilegiado el análisis de la acción estatal, y particularmente el peso de lo normativo como estructurador de las dinámicas del sistema. Más recientemente, el énfasis se ha trasladado al establecimiento de los lazos de correspondencia en- 
tre las políticas del sector y los paradigmas neoliberales en educación. Ambos enfoques operan desde lo que autores como Cavarozzi (1996) denominan "matriz estado-céntrica", con lo cual se pierde de vista el juego complejo que se establece entre los condicionantes estructurales y las diferentes estrategias y recursos que despliegan los actores. ${ }^{1}$ Un segundo obstáculo no desligado del anterior, radica - a nuestro juicio - en las insuficiencias y/o limitaciones que presenta el uso del instrumental teórico proveniente de los países desarrollados, cuando éste es aplicado al estudio de los procesos de cambio institucional en los países como Argentina, donde el gobierno de las universidades públicas resulta bastante diferente de las formas que adopta en los países europeos o en el mundo anglosajón.

En este sentido, el presente trabajo intenta avanzar en una doble línea de reflexión sobre este campo relativamente poco explorado en nuestro medio, como son los problemas de gobierno y gestión universitaria. Por un lado, se plantea intervenir y aportar al debate teórico-metodológico alrededor de los marcos conceptuales más adecuados para el estudio de estas temáticas. En esta dirección, y luego de una breve revisión de los enfoques dominantes, el trabajo presenta una primer y provisoria reformulación de las categorías de campo académico, cultura institucional y cultura política. Dichas categorías, si bien pertenecen a diferentes universos teóricos, pensamos que se justifican y pueden resultar fructíferas para el estudio de la realidad universitaria, en la medida que permiten dar cuenta de la superposición no necesariamente contradictoria de racionalidades y prácticas presentes en la producción/ reproducción académica, y a su vez, de la fuerte presencia del contexto socio-político en que se insertan.

Por otro lado, el trabajo también constituye una

${ }^{1}$ En rigor, podría señalarse un tercer tipo de estudios que muy genéricamente se pueden denominar histórico-sociales, ver: M. Mollis (1994), Universidades y Estado Nacional, ArgentinaJapón 1885-1930, Buenos Aires, Edit. Biblos, y A. Perez Lindo (1985), Universidad, política y sociedad, Buenos Aires, EUDEBA. primera puesta a prueba del instrumental presentado. En tal sentido, la caracterización de algunas lógicas y rasgos emergentes dentro del campo académico, como pueden ser el análisis de las tensiones que generan las políticas estatales al interior de la gestión y el gobierno universitario, constituirán el foco de atención de esta segunda parte. Adelantando las conclusiones, el presente trabajo postula que la emergencia de una doble línea de gestión y conducción, producto de la progresiva cristalización de nuevos actores al interior de las universidades argentinas, configuran dos fenómenos que comienzan a disputar (y a desplazar en algunos casos) el papel tradicionalmente asignado a los consejos académicos y superiores, en los procesos de toma de decisión. En síntesis, las tensiones que hoy recorren los procesos de cambio institucional son la expresión del conflicto entre las nuevas formas de gestión que incorporan las políticas oficiales y el modelo del cogobierno entre estudiantes, profesores y graduados (egresados) que instauró la Reforma Universitaria de 1918.

\section{Problemas conceptuales}

\section{a. Gobierno y organización académica}

Si la preocupación por la problemática del gobierno o la gestión, y en términos más generales del cambio universitario, tienen una presencia reciente en nuestro medio, no es éste el caso de lo que ocurre en el mundo académico anglosajón o europeo donde la vasta producción de papers y trabajos acredita el reconocimiento de un verdadero sub-campo dentro de los estudios de educación superior. En este contexto podemos identificar - grosso modo - al menos tres grandes enfoques sobre los que se asienta la gran mayoría de los trabajos; estos son: el enfoque organizacional, el enfoque burocrático y el enfoque denominado de sistema político. ${ }^{2}$

2 Para los dos últimos enfoques nos hemos basado fundamentalmente en el análisis y comentario crítico desarrollado por Pedro Krotsch (1994), em Puiggrós y Krotsch (comp.). 
Burton Clark (1983) es quizás el especialista más representativo del primer enfoque. Para este autor, el rasgo distintivo de las universidades, y que diferencia a estas instituciones de otras agencias educativas, está dada por su capacidad de producir conocimientos. Es esta característica la que hace que la disciplina y el establecimiento constituyan las formas básicas y naturales en que se estructura la organización académica. De esta manera, las tendencias que señala como la fragmentación disciplinar, la ambigüedad de los fines y los intereses de sus actores, no son más que la expresión de las lógicas que regulan la producción de conocimientos. La idea de "anarquía organizada" con que define a las universidades resume en buen parte su visión, dando cuenta a su vez de las tensiones que producen al interior de las instituciones las tendencias mencionadas.

No muy distante del planteo organizacional, los enfoques burocráticos han puesto el énfasis en la distribución de jerarquías, en el conjunto de reglas y procedimientos que orientan el funcionamiento institucional y han dado especial importancia al rol y características de los cuerpos directivos como burocracia académica. Aunque estos estudios intentan escapar a una excesiva racionalidad instrumental en sus interpretaciones (que por otro lado está inscripta en el propio modelo teórico), lo cierto es que la notable autonomía de la que gozan estas instituciones basadas en los rasgos definidos por Clark, presentan una fuerte dificultad para el análisis burocrático. Prueba de ello son las propias categorías elaboradas en el marco de este enfoque que, como "sistema débilmente acoplado" o de "acoplamiento laxo" (Weick, 1976), marcan en última instancia la distancia entre las dinámicas institucionales de la empresa o la administración estatal y las universidades.

A diferencia de los dos enfoques anteriores, el modelo de sistema político desplaza su mirada de las modalidades organizacionales hacia las características que asumen los procesos de toma de decisión al interior de las instituciones académicas (Baldridge apud Krotsch). En este sentido, la direccionalidad de las acciones o más específicamente la producción de políticas es el resultado (incierto) de la negociación y el conflicto entre una multiplicidad de actores (burócratas, grupos disciplinares o individuos influyentes). ${ }^{3}$ En nuestra opinión el éxito relativo de este enfoque radica en su ductilidad para el análisis de los problemas de gobierno y gestión tanto en sistemas universitarios estructurados de manera colegiada (modelo departamental norteamericano) como en la forma de burocracia académica (modelo europeo continental).

No cabe duda de que cada uno de los enfoques muy brevemente reseñados aporta una dimensión relevante a la hora de mirar el funcionamiento y los procesos de cambio en las instituciones de educación superior. Sin embargo, nos parece que la configuración histórica de las universidades en la Argentina presenta problemas, sino insolubles, al menos de difícil comprensión para estos enfoques. En primer lugar, el tipo de fragmentación característico de nuestras universidades, si bien se relaciona con las disciplinas y el tamaño de los establecimientos, está dada en mayor medida por la estructura jerárquica de las cátedras. La organización por cargos y dedicaciones - mayormente en carreras masivas - no sólo fragmenta sino que tiende a segmentar y estamentalizar los cuerpos docentes. Diferente es la situación en los países desarrollados donde la temprana diversificación de los sistemas (caso norteamericano) configuró dos tipos diferentes de instituciones: las centradas en la producción de conocimientos y en el post-grado, y aquellas tendientes

${ }^{3}$ En palabras de Baldridge “...dado que la elaboración y formación de políticas constituye el núcleo de la comprensión del proceso de toma de decisiones, se debe enfatizar el papel que la negociación y el conflicto juegan en relación con la participación de diversos grupos de interés. Las decisiones no son el producto de órdenes en el sentido de la perspectiva burocrática, tampoco el reconocimiento indiscutible de una autoridad respaldada en la competencia técnico-académica, sino antes bien, el resultado no esperado de compromisos entre grupos que disputan el control de recursos materiales y simbólicos que hacen a la reproducción del entramado de poder al interior de la institución" (Baldridge apud por Krotsch). 
a cubrir una demanda amplia de certificaciones profesionales. En segundo lugar, la caracterización de burocracia académica que podría asignársele a los cuerpos directivos en nuestras universidades, resulta demasiado desproporcionado, ya que en rigor son formas colegiadas (como pueden ser los consejos directivos o superiores), que resultan ser además instancias ejecutivas que delegan o encomiendan la gestión de sus decisiones a determinados miembros elegidos para tal fin (secretarios, decanos, rectores etc.). Nuevamente reaparece la distancia entre instituciones del centro y la periferia, donde en las primeras existe una fuerte tradición de profesionalización de las tareas de gestión, a comparación de la tradición "amateurista" de nuestros cuerpos directivos. En tercer lugar, aunque el enfoque de sistema político parecería ser el más adecuado para el análisis del gobierno y la gestión universitaria, pensamos que el modelo de co-gobierno que instaura la Reforma Universitaria de 1918, contiene otros aspectos que complejizan la imagen excesivamente "pluralista" (en términos de sistema político) de la organización académica que trasmite este enfoque. Podemos decir que en el co-gobierno, si bien están presentes lógicas propias de un pluralismo político (por ejemplo: las agrupaciones y tendencias) también contiene fuertes elementos corporativos (por ejemplo: los claustros). ${ }^{4}$ La complejidad mencionada en última instancia alude a la variabilidad de los componentes corporativo y competitivo pluralista que atraviesan la composición tanto de los grupos académicos, los claustros y las propias estructuras institucionales.

\section{b. Campo académico, cultura política y cultura institucional}

Este breve balance de aportes y limitaciones de los enfoques dominantes en el mundo académico

\footnotetext{
${ }^{4}$ Se denominan "claustros" a los estamentos que componen el gobierno de la universidad y que representan a los profesores, estudiantes y egresados.
}

anglosajón nos muestra por un lado, los mayores márgenes de autonomía respecto de otras esferas sociales que tienen las universidades del centro, que se evidencia en una relativa estabilidad de estas instituciones. Por otro lado, y en contraste con esta situación, las universidades argentinas, además de la modalidad específica del gobierno universitario, también presentan otro rasgo que a nuestro juicio resulta central para entender las dinámicas de cambio, como es su mayor vulnerabilidad y debilidad institucional producto de las discontinuidades políticas, y su permeabilidad a las lógicas provenientes del entorno social y político local. ${ }^{5}$

La noción bourdiana de campo y el concepto semiótico de cultura de Clifford Geertz, para explorar la cultura política y la cultura institucional en nuestras universidades, constituyen a nuestro juicio un conjunto de categorías analíticas que posibilitan dar cuenta de las especificidades locales sin perder algunos de los elementos presentes en los enfoques anteriormente comentados.

Con el concepto de campo, Bourdieu se vale de una metáfora espacial para dar cuenta no sólo de la existencia de lugares o posiciones diferentes sino que dicho espacio se estructura y funciona precisamente a partir de las relaciones que se establecen entre los ocupantes transitorios de estas posiciones. Asimismo, los componentes de lucha y disputa constituyen un rasgo esencial de todo campo, que condiciona y determina la acción y las diferentes estrategias que ponen en juego cada uno de los ocupantes de posiciones. Finalmente, la especificidad de cada campo (artístico, intelectual, político, burocrático, científico etc.) viene dada por el tipo de capital que regula dicho campo. ${ }^{6}$

${ }^{5}$ Lo anterior no significa que estemos volviendo a caer en las perspectivas estructurales antes criticadas, muy por el contrario lo que sostenemos es la necesidad de construir una mirada que, sin perder de vista las condiciones y los condicionantes macro-sociales pueda dar cuenta de las particulares configuraciones y dinámicas de las instituciones universitarias.

${ }^{6}$ La definición que se transcribe ilustra los rasgos generales de esta noción, así: “...un campo puede definirse como una red o 
Como todo concepto, el de campo nos ilumina una serie de aspectos y - como no podía ser de otra manera - nos deja en penumbra otros. Una de las críticas que se le ha realizado a esta noción, y sobretodo a la de estrategia, es la excesiva racionalidad con que se supone actúan los agentes al interior del campo, con lo cual - y pese al esfuerzo de Bourdieu por articular los comportamientos individuales/sociales al resto de los campos del espacio social - el mismo concepto tiende a acentuar las interpretaciones internistas de este espacio en particular. Es claro que Bourdieu, cuando realiza sus estudios sobre el arte, la literatura y especialmente en el campo intelectual de la sociedad francesa, aborda una realidad social altamente estructurada y fuertemente segmentada, lo cual justifica y posibilita la relativa autonomía que es constitutiva de cada campo.

Esta situación es quizás la que marca no sólo la distancia entre los países de centro y de la periferia, sino también las limitaciones del propio concepto. En nuestro caso, las universidades argentinas y en general las latinoamericanas como instituciones estatales no han estado al margen de los abatares de la inestabilidad institucional, ni tampoco de la influencia de los movimientos políticos a lo largo de su historia, muy por el contrario han estado permanentemente atravesadas por las contingencias socio-políticas nacionales. Por esta razón, una lectura ortodoxamente bourdiana no posibilita dar cuenta acabadamente de esta otra dimensión de análisis. Son esta serie de limitaciones las que habilitan una utilización de la noción de campo en un sentido bastante amplio y no necesariamente fiel al conjunto de notas con que aquí lo caracterizamos. Así, preferimos hablar indistinta-

configuración de relaciones objetivas entre posiciones. Estas posiciones se definen objetivamente en su existencia y en las determinaciones que imponen a sus ocupantes, ya sean agentes o instituciones, por su situación (situs) actual y potencial en la estructura de la distribución de las diferentes especies de poder (o de capital) [...] y, de paso, por sus relaciones objetivas con las demás posiciones (dominación, subordinación, homología etc.) (Bourdieu, 1995, p. 64). mente de campo académico o campo científico ${ }^{7}$ para enfatizar el carácter segmentado de la vida universitaria y la lógica de disputa que permanentemente atraviesan las instituciones de educación superior. Como veremos en la segunda sección, la convivencia de distintos tipos de estrategias y de recursos (capitales) con que se mueven los actores, hacen difícil ajustarse a una definición particular de campo.

Además de eso, los largos períodos de intervención y la siempre conflictiva relación con el Estado aún en los momentos democráticos, han dejado huellas profundas en el campo académico, que se manifiestan de una diversidad de prácticas y lógicas de funcionamiento (modalizándose de manera específica según disciplinas e instituciones) pero en las que es posible reconocer cierta matriz común en términos de significación. Con todas las limitaciones que luego enunciaremos, el concepto de cultura, y en especial el de cultura política y cultura institucional, nos parece que permiten dar cuenta de estas continuidades.

Como bien señala Norbert Lechner (1987), a propósito de los estudios sobre cultura política latinoamericana, las críticas principales apuntan a que es "una categoría residual que abarca de modo arbitrario, según conveniencias del caso, una multiplicidad de aspectos dispares. El empleo demasiado extensivo y poco riguroso del término reduce su valor informativo". A su vez, al ser una temática abordada tanto desde las corrientes marxistas como desde el funcionalismo, se ha tendido a amalgamar, sin una clara y necesaria distinción teórica, cuestiones como las ideologías de clase, las orientaciones de valores y las tendencias de la opinión pública. Pese a la justeza de algunas de estas críticas, lo cierto es que los estudios que toman

7 Vale aclarar que la distinción entre ambos campos no es menor, ya supone espacios diferenciaciados como son la universidades y la ciencia. Bourdieu en su clásico Homo Academicus (1990) ha desarrollado la especificidad del primer campo, y ha dedicado numerosos artículos al segundo: ver, entre otros, P. Bourdieu (1994), "El campo científico", Revista Redes, n. 2, Universidad Nacional de Quilmes. 
como objeto las transformaciones culturales han abierto un campo de problemas no reducibles a las explicaciones en términos solamente socio-económicos o ideológico-valorativos. En el marco de este somero balance de críticas y potencialidades de los conceptos de cultura política o cultura institucional, resulta claro que el punto inicial de la discusión se relaciona con el propio concepto de cultura.

En este sentido, y apoyándonos en la perspectiva geertziana, podemos decir que la cultura no constituye un agregado de ritos, creencias o mitos (sean estos primitivos o modernos) sino que es una trama o estructura de significaciones más amplia donde cobran sentido esas unidades menores que el investigador recorta. Este concepto semiótico de cultura que postula Geertz, ${ }^{8}$ es el que le permite avanzar sobre campos que fueron tradicionalmente objeto de otras disciplinas como la historia, la sociología, ciencia política y que en nuestro caso nos habilitan para explorar el universo simbólico universitario en términos de cultura política. ${ }^{9}$

Retomando a Lechner, podemos decir que la cultura política incorpora la dimensión temporal ya que la misma noción alude a la consolidación de determinadas pautas que se transmiten en largos procesos de

\footnotetext{
${ }^{8}$ Refiriéndose al concepto de cultura, Geertz lo define de la siguiente forma: "Entendido [la cultura] como sistema de interación de signos interpretables (que, ignorando las acepciones provinciales, yo llamaría símbolos), la cultura no es un entidad, algo a que pueden atribuirse de manera causal acontecimientos sociales, modos de conducta, instituciones o procesos sociales; la cultura es un contexto dentro del cual pueden describirse todos esos fenómenos de manera inteligible [...]" (Geertz, 1995, p. 27).

${ }^{9}$ De este autor tomamos la siguiente definición de cultura política que, como se observará, se distancia del naturalismo antropológico y del análisis político institucional clásico: “Aquí, cultura no es ni culto ni usanza, sino que son las estructuras de significación en virtud de las cuales los hombres dan forma a sus experiencias; $y$ la política no es aquí golpes de estado ni constituciones, sino que es uno de los principales escenarios en que se desenvuelven públicamente dichas estructuras. Una vez reformuladas así política y cultura, determinar la relación que hay entre ellas es una empresa práctica y no ciertamente modesta" (Geertz, 1995, p. 262).
}

socialización, y que actúan sobredeterminando la propia acción de los actores. Correlativamente la noción de cultura institucional apunta a articular de modo dinámico el cruce entre historia institucional y cultura académica, entendida esta última como trama de significados derivados, o mejor dicho, que tienen en su base - aunque no exclusivamente - una pertenencia disciplinar. La dificultad que se nos presenta, y que en buena medida se expresa en una deliberada ambigüedad de estos conceptos, es que la realidad universitaria argentina se despliega en formas bastante híbridas donde se superponen simultáneamente, y no necesariamente de modo contradictorio, aspectos de la cultura política, de la cultura institucional y de la cultura académica. Este rasgo de sincretismo es quizás el que nos obliga a diferenciarnos de los marcos de análisis dominante en el mundo desarrollado donde estas esferas están mucho más separadas y delimitadas. ${ }^{10}$

Sintetizando lo hasta aquí expuesto, decíamos que la noción de campo (científico y/o académico) nos brinda un marco de análisis, que sin abandonar la incidencia de las determinaciones globales, posibilita insertar y dar cuenta del sentido de las acciones (de grupos o individuos) en términos de formas particulares de racionalidad. A su vez, el concepto geertziano de cultura nos permitió incorporar a nuestro marco de referencia la dimensión temporal en que se transmiten, consolidan o transforman determinadas estructuras de significación, que en nuestro caso no son reductibles solamente a la pertenencia disciplinar o institucional y que, como se verá en la próxima sección, se explican por la permeabilidad de las instituciones universitarias a las lógicas de la cultura política nacional.

10 Aclaramos que no decimos que en las universidades en USA y Europa no se presente la misma dificultad en precisar aspectos de la cultura institucional y de la cultura académica (ver Clark, 1983). Lo que deseamos enfatizar es que en el caso de Argentina, las instituciones universitarias y el campo científico no tienen el grado de consolidación que poseen los paises del centro y que, como desarrollaremos mas adelante, se encuentran permeadas por pautas más propias de la cultura política. 
De esta manera, la reformulación de las nociones de campo científico/académico, cultura institucional y cultura política, y la tendencia a la superposición de éstas, recuperan un rasgo básico para entender la realidad universitaria argentina, como es la hibridez socio-cultural de sus instituciones académicas. Finalmente, el conjunto de notas contenidas en estas nociones permiten a su vez aproximarnos a la comprensión de las nuevas y viejas identidades académicas (entendidas éstas como un proceso de constitución de subjetividades) en la medida que articulan el aspecto objetivo/estructural y el subjetivo/simbólico desde una dimensión histórica.

\section{Tendencias emergentes en el gobierno y la gestión académica}

\author{
a. Estado, Universidad y campo \\ académico en la Argentina
}

En las últimas tres décadas la relación entre Universidad y Estado en la Argentina se estructuró alrededor de un patrón básicamente inestable que alternó períodos de fuerte control y represión física e ideológica durante los regímenes políticos autoritarios, y momentos de mayor apertura y permisividad en los regímenes democráticos. ${ }^{11}$ En general este comportamiento también se articuló en materia de financiamiento a tendencias que combinaron el congelamiento y/o restricción presupuestaria con el estímulo al sector privado, por un lado; y una tendencia incremental de los fondos públicos para el sector, por otro (Brunner, 1993). En este contexto, un elemento de continuidad

${ }^{11}$ Vale aclarar que si bien la inestabilidad política fue una constante a nivel regional en las décadas mencionadas, la actitud asumida por los distintos regímenes autoritarios frente a las universidades no resultó homogénea, al contrario es posible observar la configuración de diferentes estrategias como por ejemplo las desarrolladas en los casos de Brasil y Chile. Para una visión comparativa de la relación Estado-Universidad en latinoamerica resulta muy esclaracedor el trabajo de Daniel Levy (1995). (por lo menos hasta mediados de los 80') fue la incapacidad o imposibilidad de parte de los transitorios ocupantes del estado, de generar proyectos de cambio, que vayan más allá de la actitud represora o benevolente frente a las universidades, y por ende, de estructurar otro patrón de relación sobre nuevos criterios.

A nivel de la base del sistema, este movimiento pendular se manifestó en la consolidación de una serie de comportamientos y estrategias, variables según los campos disciplinares y las dimensiones de cada universidad. En algunos casos, acentuó o exacerbó las tendencias corporativas como puede ser en el caso de las llamadas ciencias duras, mientras que en otros, la mayor debilidad en cuanto a su inserción institucional y al grado de profesionalización de sus cuerpos docentes acentuó tendencias a la atomización académica y a la incentivación de comportamientos individualistas, como por ejemplo en las ciencias sociales. Estas variaciones explican en parte las diferentes capacidades tanto de resistencia en los momentos de restricción política como de recuperación y reposicionamiento en aquellos de apertura democrática (Suasnábar et al., 1997).

Los largos períodos de intervención de las universidades nacionales han consolidado para este sector una fuerte sensación de recelo y desconfianza hacia el Estado, del cual solamente se demanda que provea los recursos necesarios para el funcionamiento institucional. Por esta razón, la idea de autonomía, tan cara a la comunidad académica, ha mantenido una fuerte presencia en el discurso universitario pero enfatizando su costado en términos de libertad negativa (no injerencia estatal) y menos - como veremos más adelante - su costado positivo, como autogobierno.

De esta manera, las características apuntadas sobre las relaciones entre Estado y Universidad fueron sedimentando, y en muchos casos, cristalizando determinadas estructuras de significación que atraviesan y moldean las diferentes culturas disciplinares e institucionales. Aunque todavía son escasos los trabajos empíricos enfocados en esta dirección (Vessuri, 1992; Oteiza y otros, 1992) y que permitirían dar cuenta más 
acabadamente de las huellas dejadas en las comunidades académicas por los períodos precedentes; es evidente que en coyunturas de cambio como las actuales emergen una serie de comportamientos que van más allá de la simple adaptación o rechazo a las políticas implementadas. En nuestra opinión, las razones que explican estas actitudes hay que buscarlas en los elementos constitutivos de la cultura académica y en los efectos desestructurantes sobre ésta, que las políticas oficiales incorporan en términos de nuevas formas de regulación.

b. Los cambios en las formas de regulación social

Con la reinstauración de la democracia en los años 80", y luego de la etapa llamada de "normalización", las universidades argentinas retomaron las pautas básicas de funcionamiento marcadas por el modelo reformista. Sin embargo, y pese a que este cambio abrió un espacio de mayor protagonismo del campo académico, el patrón de funcionamiento antes descripto se prolongó en líneas generales durante los primeros años del gobierno democrático. No es sino hasta comienzos de los años 90', con la creación de la Secretaría de Políticas Universitarias (SPU) en el marco del Ministerio de Educación, donde se empieza a operar un cambio en las políticas estatales para el sector, que de alguna manera expresan propuestas ampliamente difundidas por los organismos de crédito internacional, y aplicadas en la década anterior por diferentes gobiernos de la región.

El cambio en las formas de financiamiento y la incorporación de mecanismos de evaluación configuran dos dispositivos en los que se asientan estas propuestas de reestructuración de las universidades. Por un lado, el modelo de distribución presupuestario se dualiza rompiendo la tendencia incremental señalada, ya que se congelan las partidas destinadas al funcionamiento, y se incorporan los llamados programas especiales, ${ }^{12}$ los cuales constituyen fondos destinados a rubros es-

${ }^{12}$ Nos referimos básicamente al conjunto de programas que en los últimos años viene desarrollando la Secretaría de Políticas pecíficos (investigación, grado y post-grado) sujetos a la competencia inter-institucional. Por otro, el discurso y la práctica de la evaluación instala una nueva modalidad de control sobre las instituciones universitarias, que se manifiesta en la creación de organismos nacionales como la Comisión Nacional de Evaluación y Acreditación Universitaria (CONEAU) y la introducción en la mayoría de los programas especiales de prácticas como los comités de pares para su evaluación y/o seguimiento.

Los resultados de estas políticas hasta el presente han sido por lo menos contradictorios, cuando no perversos. Un ejemplo de ello son las políticas en el área de investigación: si bien es cierto que algunos programas han permitido mejorar las condiciones para el desarrollo de esta actividad, también tienen como contracara las tendencias a la fragmentación y elitización del sector. ${ }^{13}$ A su vez, el carácter centralizado de estas políticas combinadas con la evaluación de pares, que aspiraba a una mayor objetividad en la asignación de recursos, ha degenerado en mecanismos altamente burocráticos y no necesariamente más transparentes.

Hablamos de un cambio en las formas de regulación social (Popkewitz, 1992) ${ }^{14}$ en la medida que estos dispositivos no sólo reestructuran el patrón his-

Unversitarias como pueden ser: el Programa de Incentivos y la categorización de investigadores, el Sistema de Acreditación de postgrados, el FOMEC (Fondo para el Mejoramienteo de la Calidad) con créditos del Banco Mundial y recientemente el PROFIDE, que es un fondo que financia proyectos específicos asignado de manera competitiva entre las universidades.

${ }^{13}$ En un trabajo anterior (Suasnábar, 1995) hemos analizado como las políticas de incentivos docentes y de categorización de investigadores profundizan las tendencias a la fragmentación del cuerpo docente, situación que se manifiesta en la ampliación de la brecha entre transmisión y producción de conocimientos, y en la segmentación y elitización al interior del sector de docentes-investigadores. Asimismo, señalabamos que la progresiva expansión de estos procesos en la base tienden a acentuar las diferencias a nivel de departamentos, facultades y universidades.

${ }^{14}$ Para Popkewitz el estudio de la reforma educativa - como práctica y como discurso - constituye una parte de un estudio mayor 
tórico de funcionamiento (introduciendo nuevas "reglas del juego" en la relación Estado-Universidad), sino que fundamentalmente tienden a un fuerte proceso de resocialización, y a la vez, de disciplinamiento de los sujetos y sus prácticas académicas. En una línea de pensamiento foucaultiana de larga duración, diríamos que las formas precedentes de control sobre las universidades se centraron más en las instituciones que en las personas, y más en base a la coerción que en la generación de consensos. ${ }^{15}$ De este modo, las categorías de investigador, las certificaciones de post-grado, la cantidad de papers en revistas con referato, entre otros "indicadores" configuran la nueva cuadrícula que clasifica jerárquicamente a los individuos y que, a la vez, desplazan los viejos códigos de reconocimiento académico.

Este proceso de "panoptización" de vida académica - aún en estado embrionario - tiene como un segundo efecto la modificación de las lógicas estructurantes de los campos académicos, en la medida que supone, por un lado, una estandarización de los capitales puestos en juego y, por otro, la generación de nuevas estrategias de disputa. Al contrario de las expectativas oficiales, que buscan en la homogeneidad de criterios mayores niveles de previsibilidad, racionalidad y objetividad, los nuevos mecanismos han producido más incertidumbre que certezas. Así, a

de los procesos de regulación social. Con este concepto este autor intenta avanzar en una mirada socio-histórica más integral que dé cuenta tanto de los aspectos globales de los mecanismos de control pero a su vez, de las "formas específicas y regionales" a través de las cuales el poder afecta las instituciones y a los propios individuos. Influenciado por el pensamiento post-moderno y por las corrientes post-estructuralistas, el concepto de regulación social reformula la noción foucaultiana de "gubernamentalidad" e integra las formulaciones críticas realizadas al reproductivismo estructuralista, en particular a los conceptos de control social, poder y dominación.

${ }^{15}$ Quizás una excepción a esta tendencia en la historia reciente de la universidad argentina haya sido el período 1958-66, donde el clima de la modernización desarrollista articuló, no sin contradicciones, a la élite política con bastos sectores de la intelectualidad universitaria (ver Terán, 1991 y Sigal,1991). excepción de las disciplinas altamente globalizadas con circuitos fuertemente formalizados como la física, la astronomía o la biología (y que en alguna medida operan como parámetro de estas políticas), el resto de los campos, como las ciencias sociales, las ciencias de la salud y la totalidad de las carreras profesionales y artísticas, plantean infinidad de problemas a la hora de definir y evaluar la calidad de sus prácticas específicas.

Si el componente de lucha es una de las características de todo campo, en contextos que tienden a una polarización global del sector con integrados y excluidos, y a una segmentación dentro del primer grupo, las disputas por la legitimidad y el reconocimiento adquieren formas hobbesianas, ${ }^{16}$ en la medida que se exacerban los componentes de individualismo, corporativismo y elitismo propios del trabajo académico. Las culturas académicas como condensación heterónoma de nuevas y viejas tendencias, nos brinda un marco de referencia (donde se insertan las prácticas del autogobierno) para poder entender las lógicas que están regulando el comportamiento institucional.

\section{c. Gobierno universitario, campo académico y cultura política}

La multiplicidad de tensiones y conflictos que generan las transformaciones en la base del sistema tienen su expresión y se manifiestan en las lógicas de gobierno de las instituciones universitarias. De esta manera, los comportamientos de los actores del campo académico que generan las nuevas formas de regulación, se encuentran atravesados por las formas

${ }^{16} \mathrm{El}$ caso de los historiadores de la UNLP analizado en un trabajo reciente (Suasnábar y otros, 1998) constituye una buena aproximación a las prácticas y lógicas que aquí caracterizamos como "hobbesianas". Si bien los resultados de esta investigación son provisorios, las críticas al sistema de categorización y el recelo frente a las nóminas de evaluadores en otras disciplinas, parecerían indicar una mayor amplitud de estos comportamientos. 
de gobierno cristalizadas en la cultura institucional, y por estilos de gestión solamente explicables por la influencia de aspectos propios de la cultura política. Las características mencionadas, sin embargo no constituyen un rasgo posible de ser aplicable al conjunto de las universidades nacionales, ya que la propia constitución histórica del sistema plantea variaciones entre las universidades grandes de larga tradición (como Buenos Aires, La Plata y Córdoba), aquellas creadas en los años 60-70 (en general radicadas en las provincias) y las de reciente creación como las del conurbano bonaerense. En este sentido, los comentarios que siguen resultan más ajustados para las universidades del primer grupo aunque, como muestran trabajos recientes (García de Fanelli, 1997), parecería indicar la presencia de nuevas articulaciones entre cultura institucional y cultura política en las instituciones del último grupo, lo cual sugeriría que, aunque los argumentos a desarrollar no tengan una validez general, sí lo tenga el modelo de análisis propuesto.

Un primer aspecto a señalar es que en realidad gran parte de las tensiones no son nuevas, sino que son constitutivas del propio modelo reformista. Así, las ideas-fuerza del co-gobierno y el sentido positivo de la idea de autonomía como autogestión institucional recuperan metafóricamente el modelo de la república democrática donde el "pueblo" está representado por los claustros, mientras que los consejos superiores y académicos adquieren simultáneamente la forma de "parlamentos" y de "gobierno" universitario. Esta forma de concebir el "demos" universitario se asienta en una relación tácita entre democracia, saber y autoridad. Resumiendo el argumento reformista, diríamos que el sentido democratizador que plantea la representación por claustros, supone que las identidades de los actores se conforma y configura solamente a partir de su posición diferencial en la organización académica, y que asimismo, basa su representatividad en un saber o competencia legítima. De tal forma, la autoridad de las instancias directivas de la institución intenta sintetizar democracia y saber académico.

Es evidente que este modelo típico ideal del reformismo presenta las misma críticas y limitaciones que se le pueden realizar a toda concepción democrá- tica liberal de gobierno: individualismo metodológico, igualitarismo formal e ideal del bien común. Asimismo, este ideario lejos de cumplirse, en la práctica se asemeja más a una democracia "restringida" regulada corporativamente y legitimada por el voto censitario, que a una verdadera democracia liberal. Lo paradójico de esta situación es la recurrencia en el discurso de casi todos los actores académicos a esta metáfora del "demos" universitario.

La expansión matricular, la diversificación y complejización de los campos disciplinares y profesionales, la segmentación y jerarquización del cuerpo docente, son algunos de los procesos que también cuestionan la validez y eficacia del modelo de gobierno reformista, y que por otra parte, ya estaban presentes en los 60' y 70'. No obstante, la histórica inestabilidad institucional de las universidades parecería que ha operado reafirmando en el imaginario universitario este modelo, el cual, pese a responder cada vez menos a la realidad, al menos - se pensaba - sirve de freno a toda intervención externa y deja abierta la posibilidad de su propia reformulación. Los más de quince años transcurridos desde la normalización universitaria post-dictadura en 1983-84 mostraron no sólo lo limitado de estas expectativas, sino fundamentalmente dejaron en evidencia la dificultad, por parte de la comunidad universitaria, de generar proyectos autónomos de cambio.

Pero las razones de esta incapacidad de autorenovación institucional no derivan solamente de las limitaciones del modelo reformista de gobierno. Las prácticas resultantes de la combinación de estructuras de significación de la cultura académica y la cultura política en la base, en un esquema de gobierno regulado por "reglas de juego" propias de la política, generó condiciones para el desarrollo en los niveles intermedios (facultades y departamentos) y superiores de conducción (rectorados) de formas clientelares que vuelven opacas las mediaciones de la representación académico-estamental. ${ }^{17}$ Las redes de intermediación

${ }^{17}$ Tal vez se nos pueda criticar la pertinencia del término “clientelismo" para caracterizar ciertas prácticas en la universidad. 
que supone todo clientelismo adquieren en la universidad una forma mixta donde el interés corporativo (disciplinar y/o profesional) se combina con los intereses de grupo (investigadores formados, becarios, estudiantes, docentes no investigadores etc.), delineando alianzas verticales y horizontales, esto es, que se expresan tanto en el plano de la diferenciación de campos académicos como en el plano de la jerarquía entre y al interior de los campos. En este contexto de fragmentación de intereses, la creciente partidización de las estructuras de gobierno no es más que el resultado del peso que adquieren las redes construidas sobre otra fuente de identidad como es la políticopartidaria. Si bien la cristalización de estos estilos de conducción y gestión posibilitó un cierto nivel de integración institucional frente al Estado, los mismos resultaron ineficaces para evitar la tendencia a la ingobernabilidad interna, y mucho menos para la generación de proyectos de cambio.

Así como las nuevas formas de regulación desestructuran las anteriores identidades académicas y las reconfiguran sobre nuevos criterios, las políticas estatales en curso también comienzan a modificar las lógicas de gobierno. Un primer rasgo es la emergencia de una doble línea de gestión y conducción que disputa la direccionalidad de las instituciones universita-

Como plantea Javier Auyero (1997), el clientelismo político alude a un tipo de relación de dominación entre agentes donde unos “ [...] le dan su apoyo y votos [a otros] presumiblemente a cambio de favores, servicios y otros bienes no materiales" (p. 179). Como señala el autor, la presunción es parte del problema empírico que tienen los estudios sobre esta temática, pese a ello, el clientelismo posibilita dar cuenta del papel que juegan las redes de intermediación en la esfera política en la relación representantes-representados. Trasladado al ámbito científico y académico, tanto Kuhn como Bourdieu han aportado a develar el carácter presuntamente “desinteresado" que estructuran las prácticas en la ciencia y en la academia. Pensamos que hablar de "formas clientelares" en la universidad nos permite dar cuenta de un rasgo que, si bien no podemos afirmar ni demostrar que sea lo dominante, al menos dá cuenta de prácticas posibles en el marco del modelo reformista de gobierno. rias. Por un lado, los consejos académicos y superiores, que como vimos, al estructurarse por este mix de lógica política y lógica académica, se vuelven lentos e ineficaces para la definición de políticas (problema del consenso) y para operativizar medidas (problema de la eficacia). Por otro lado, las nuevas estructuras (denominadas genéricamente "unidades ejecutoras") que los programas de la SPU demandan para su implementación, aunque formalmente dirigidas a la coordinación y seguimiento "técnico", empiezan a constituirse en instancias de gobierno paralelo.

Estructuradas desde una racionalidad burocrática y altamente centralizada, e insertas a nivel de facultades y universidad (y en muchos casos en departamentos), las nuevas estructuras se integran según las disciplinas: por "académicos reconocidos", por gestores "políticos" y en los menos por grupos colegiados; pero en todos los casos avanzan en definiciones propias de los organismos del co-gobierno.

La dualización de los ámbitos de toma de decisiones también dá cuenta de un segundo rasgo que es la cristalización de nuevos actores constituidos sobre la base de otras fuentes de poder. A diferencia de períodos anteriores, donde los cargos de gestión en el nivel central (ministerio) eran ocupados por sectores provenientes del campo político, los programas de la SPU han demandado la generación de una burocracia académica de altas calificaciones. Dotada de abundantes recursos, el staff ministerial priorizó una estrategia más intervencionista que discursiva, ${ }^{18}$ donde la producción de información estadística respecto del sistema global (censo de estudiantes, estadísticas de uni-

${ }^{18}$ Una somera revisión de las publicaciones y documentos ministeriales muestra que, a excepción del texto de la Ley de Educación Superior sancionada en 1995, hay una casi inexistencia de un discurso legitimador explícito. En realidad, la SPU instala una agenda de debate a partir de la producción de información y la implementación de los programas especiales, produciendo un efecto de parcialización de las temáticas donde se discute más el cómo de la implementación o no de las propuestas y menos el modelo global al que se quiere arribar. 
versidades nacionales, la categorización y acreditación de post-grado etc.), y en menor medida la investigación específica, se combina con la implementación y gestión de los programas especiales. Paralelamente, la legitimación de "mandarinatos académicos" en casi todas las disciplinas a partir de la confección de bancos de evaluadores y de la conformación de comisiones para acreditación de post-grados, entre otras medidas, constituyen una redistribución y desplazamiento del poder de decisión hacia estos actores que, si bien no es nueva, en el marco de estas políticas disputan y desafían la racionalidad de las acciones emanadas desde los consejos académicos y superiores. ${ }^{19}$

\section{Un cierre con final abierto}

Resumiendo los principales argumentos hasta aquí planteados, decíamos que las tensiones que introducen las nuevas formas de regulación están reconfigurando las identidades académicas, la estructura de los campos disciplinares y las culturas institucionales. Asimismo señalábamos que las características y limitaciones del modelo de gobierno reformista agregaban, en este contexto, un segundo núcleo de tensiones (dualización de las líneas de conducción y emergencia de nuevos actores) que se manifiesta en la convivencia contradictoria de formas y estilos de gobierno y gestión (cultura política). El esfuerzo por homogeneizar las políticas de modernización universitaria, finalmente, produce un efecto contrario, ya que las propuestas y acciones oficiales son resignificadas desde la particular matriz institucional, produciendo resultados altamente heterogéneos.

Por esta razón, caracterizar una declaración, una medida o una política específica, como de resistencia, adaptación o de transformación, nos dice poco si no se toman en cuenta las tendencias y procesos descriptos. Así, como Popkewitz nos advierte sobre la propensión

${ }^{19} \mathrm{El}$ ejemplo del FOMEC resulta ilustrativo de las tensiones y contradicciones que instalan esta doble línea de gestión y los nuevos actores. de asimilar reforma a cambio, y a asignarle a éste un sentido positivo per se, pensamos que la dupla resistencia/adaptación no necesariamente debe asociarse a consignas como: "la defensa de la universidad pública, gratuita, autónoma y democráticamente co-gobernada", ni tampoco a "la aceptación de las propuestas neoliberales dictadas por los organismos internacionales". En este sentido, la capacidad de los cuerpos académicos y de las instituciones universitarias de generar proyectos autónomos de cambio, parecería estar más relacionada con el grado de auto-conocimiento de las especificidades y lógicas que la atraviesan que por la aceptación u oposición a las medidas estatales. En todo caso, estas últimas constituyen parte de los condicionantes estructurales que marcan los límites a la acción de los sujetos, pero esto no supone que dicha acción esté predeterminada.

Aunque a grandes trazos los dispositivos de regulación han comenzado a transformar la fisonomía de las universidades argentinas, el proceso de modernización (como prefieren denominarlo algunos especialistas) recién está en marcha. Por ende, aún es difícil predecir la direccionalidad y sentido de los cambios. Pero quizás este ejercicio de futurología se vuelve aún más complejo, si como intentamos desarrollar en este trabajo, la reconfiguración de las identidades académicas a que llevan estas políticas sólo puede ser pensada como el resultado complejo y contradictorio de esta superposición de cultura institucional, disciplinar y política, expresada (entre otras formas) en las tomas de posición y acciones de gobierno universitario.

CLAUDIO SUASNÁBAR, mestre pela Faculdad Latinoamericana de Estudios de Maestría en Educación en la FLACSO, sede Argentina, é professor e investigador da Universidad Nacional de La Plata. Publicou recentemente: Políticas de modernización universitaria y cambio institucional, em colaboração com Guilhermina Tiramonti e Viviana Seoane (La Plata, Argentina, UNLP, Faculdad de Humanidades y Ciencias de la Educación, 1999, Serie Estudios e Investigaciones n. 38); "Revista Perspectiva Universitaria: voces decidentes en dictadura", em obra de Carolina Kaufman; Educación y dictadura: universidade y grupos académicos argentinos, 1976- 
1983 (Madrid: Miño y Dávila Ed., 2001, Tomo 1); “La reforma educativa nacional: en busca de una interpretación, em colaboração com Guilhermina Tiramonti, em Modernización educativa en los 90': el fin de la ilusión emancipadora? (Buenos Aires, Flacso \& Temas Grupo Editoraila, 2001).E-mail: csuasnab@netverk.com.ar

\section{Referencias Bibliografícas}

AUYERO, Javier, (comp.) (1997). Favores por votos? Estudios sobre clientelismo político contemporáneo. Buenos Aires: Losada.

BOURDIEU, Pierre, (1997). Para una ciencia de las obras. In: Razones prácticas. Sobre la teoría de la acción. Barcelona: Anagrama.

BOURDIEU, Pierre, (1995). La lógica de los campos. In: Repuestas; por una antropología reflexiva. México: Grijalbo.

BRUNNER, José Joaquin, (1993). Evaluación y financiamiento de la educación superior en América Latina: bases para un nuevo contrato. In: COURARD, H. (comp.). Políticas comparadas de educación superior en América Latina. Santiago de Chile: FLACSO.

CAVAROZZI, Marcelo, (1996). El agotamiento de la matriz estadocéntrica y la emergencia de la sociedad de mercado 1983-1996". In: Autoritarismo y democracia (1955-1996). La transición del Estado al Mercado en la Argentina. Buenos Aires: Ariel.

CLARK, Burton, (1983). El sistema de educación superior. una visión comparativa de la organización académica. México: Nueva Imagen.

GARCÍA DE FANELLI, Ana María, (1997). Las nuevas universidades del conurbano bonaerense: misión, demanda externa y construcción de un mercado académico. Buenos Aires: Doc. CEDES/117, Serie Educación Superior.

GEERTZ, Clifford, (1995). La interpretación de las culturas. Barcelona: Gedisa.
KROTSCH, Pedro, (1994). Organización, gobierno y evaluación universitaria. In: PUIGGRÓS, KROTSCH (comp.). Universidad y evaluación. Estado del debate. Buenos Aires: IEAS/Aique.

LECHNER, Norbert, (1987). Presentación. El nuevo interés por la cultura política. In: LECHNER, N., (comp.). Cultura política y democratización. Buenos Aires: CLACSO.

LEVY, Daniel, (1995). La educación superior y el Estado en Latinoamérica. Desafíos privados al predominio público. México: M.A. Porrua.

OTEIZA, Enrique y otros, (1992). La politica de investigación cientifica y tecnológica argentina; historias y perspectivas. Buenos Aires: CEAL.

POPKEWITZ, Thomas, (1992). Sociología Política de las reformas escolares. Madrid: Morata.

SIGAL, Silvia, (1991). Intelectuales y poder en la década del sesenta. Buenos Aires: Ed. Punto Sur.

SUASNÁBAR, Claudio, (1995). Situación del sector docente universitario y las transformaciones del mercado académico. In: Revista Propuesta Educativa, Buenos Aires, FLACSO/Miño y Dávila, $n^{\circ} 13$, dic.

SUASNÁBAR, Claudio, SEOANE, Viviana, DELDIVEDRO, Vanesa, (1998). Modelos de articulación académica: cultura e identidad de los docentes-investigadores de la UNLP. Revista Propuesta Educativa, Buenos Aires, FLACSO/Novedades Educativas, $n^{\circ} 18$, jun.

TERÁN, Oscar, (1991). Nuestros años sesenta; la formación de la nueva izquierda intelectual argentina 1956-1966. Buenos Aires: Ed. El Cielo por Asalto.

VESSURI, Hebe, (1992). Las ciencias sociales en Argentina: diagnóstico y perspectivas. In: OTEIZA, Enrique, (comp.). La política de investigación científica y tecnológica Argentina. Historias y perspectivas. Buenos Aires: CEAL.

WEICK, Karl, (1976). "Educacional organization as boosely coupled sistems". In: Ashe Reader: an organization and governance in Higher Education. New York: John Wylly. 
work and school education. The discourse of the subjects of the research reveals the conflict between the formation required for cooperative agricultural work and the model of basic education on which rural education is based.

\section{Isabel Lelis}

Profissão docente: uma rede de histórias

Este artigo tem o objetivo de analisar a identidade social do magistério das séries iniciais do ensino fundamental, forjada em processos de socialização familiar, escolar e profissional. Através do recurso às histórias de vida de treze professoras, foi possível perceber que muitos são os significados atribuídos ao trabalho docente. Recorrendo ao aporte da sociologia de Pierre Bourdieu, o trabalho permitiu compreender a constituição de habitus para a docência, as estratégias desenvolvidas pelas professoras para a conquista de títulos escolares, os modos de entrada na profissão, o peso da formação prévia e continuada nas formas como as professoras vivem e representam o trabalho que realizam. Considerando que a representação social do magistério, no contexto de uma escola de massa, foi alterada, o estudo acabou por problematizar imagens de passividade, negligência e incompetência que têm sido atribuídas às professoras pelas burocracias educacionais e agências governamentais.

The teaching profession: a webs of stories

This article proposes to analyse the social identity of teachers in the first series of primary education, forged through processes of professional, school and family socialization. Using the life stories of thirteen women teachers, the article discusses the significance attributed to the teaching profession. Taking Pierre Bourdieu's sociology as reference, the study explores the constitution of the habitus in teachers, the strategies they use to achieve academic qualifications, admission to the profession and the weight of initial and continued training in the way teachers experience and represent their work. This study challenges the images of passivity, negligence and technical incompetence that are attributed to teachers by educational authorities and governamental agencies, by recognizing that, at a time in which a massification of school is occuring, the social representation of teaching has been modified.

\section{Claudio Suasnábar}

Resistência, mudança e adaptação nas universidades argentinas: Problemas conceptuais e tendências emergentes no governo e na gestão acadêmica

$\mathrm{O}$ artigo propõe-se a fazer uma contribuição ao debate teóricometodológico dos referenciais conceptuais mais adequados para o estudo dos problemas do governo e da gestão universitária na Argentina.

Este trabalho também constitui uma primeira posta à prova do instrumental apresentado. São analisadas as tensões que geram as políticas estatais na gestão acadêmica. Considerase que a emergência de uma dupla linha de gestão e condução na institucionalização de novos atores nas universidades argentinas configuram os dois fenômenos que disputam o papel historicamente atribuído aos conselhos acadêmicos. Essas tensões, que hoje percorrem os processos de mudança institucional, expressam o conflito entre as novas formas de gestão e o modelo de co-governo que instaurou a Reforma Universitária de 1918.
Resistance, change and adaptation in argentine universities: concept problems and tendencies emerging from academic government and management

This article aims to contribute to the theoretical and methodological debate on the most suitable conceptual frameworks for studying the problems of government and management in Argentinian universities. The paper also constitutes a first test of the instruments presented therein. Tensions generated by state policies on academic management are also analysed. The article considers that the emergence of a double line of manegement and condut of the institution of new actors in Argentinian universities constitute the two phenomena which dispute the role historically attributed to academic councils. These tensions, which underlie processes of institutional change the, are the expression of conflicts between new forms of management and the model of cogovernment that established the Argentinian University Reform of 1918.

Afrânio Mendes Catani, Denice Bárbara Catani e Gilson R. de M. Pereira

As apropriações da obra de Pierre Bourdieu no campo educacional brasileiro, através de periódicos da área

A partir de pesquisa realizada em 20 periódicos especializados em educação, editados entre 1971 e 2000, são analisadas as formas de apropriação da obra de Pierre Bourdieu no campo educacional brasileiro. O conjunto dos 355 artigos publicados nesses periódicos que fazem referências ao sociólogo constitui o corpus básico para a análise das peculiaridades das interpretações brasileiras do autor. 